\title{
Correction to: Evaluation of pharmacokinetic models of intravenous dexmedetomidine in sedated patients under spinal anesthesia
}

\author{
Shinju Obara ${ }^{1}$ (D) Tsuyoshi Imaizumi ${ }^{2} \cdot$ Takahiro Hakozaki $^{2} \cdot$ Atsuyuki Hosono $^{3} \cdot$ Yuzo Iseki $^{2} \cdot$ Norie Sanbe $^{3}$. \\ Masahiro Murakawa ${ }^{3}$
}

Published online: 27 April 2018

(c) Japanese Society of Anesthesiologists 2018

Correction to: J Anesth (2018) 32:33-40

https://doi.org/10.1007/s00540-017-2424-1

Inadvertently, the reference [8] was published incorrectly in the original publication of the article. The correct reference [8] is provided below:

8. Hannivoort LN, Eleveld DJ, Proost JH, Reyntjens KMEM, Absalom AR, Vereecke HEM, Struys MMRF. Development of an optimized pharmacokinetic model for dexmedetomidine in healthy volunteers. Proceedings of 23rd annual meeting of the International Society for Anaesthetic Pharmacology. 2014. https://www.isaponline.org/applicatio n/files/3714/7699/9952/23_ISAP14_Abstract_Hannivoort .pdf. Accessed 27 Mar 2018.

In addition, the number of samples of the model in Table 1 is 379 , not 408 . The V1 of the Talke model in the same table is 16.6 , not 16.57 .
Shinju Obara

obashin99@gmail.com

1 Surgical Operation Department, Fukushima Medical University Hospital, 1 Hikarigaoka, Fukushima,

Fukushima 960-1295, Japan

2 Department of Intensive Care Medicine, Fukushima Medical University Hospital, 1 Hikarigaoka, Fukushima, Fukushima 960-1295, Japan

3 Department of Anesthesiology, Fukushima Medical University School of Medicine, 1 Hikarigaoka, Fukushima, Fukushima 960-1295, Japan 